\title{
Modelos de satisfacción: fundamentación teórica y criterios de aplicación
}

\section{Models of satisfaction: theoretical foundation and application criteria}

Fernando Haro Carrillo

Nelson Córdova Rosas

Tomás Chong Qui

Universidad Santa María, Ecuador

Autor para correspondencia: tbchongqui@gmail.com, fharoc@usm.edu.ec, ncordova@usm.edu.ec

Fecha de recepción: 30 de Agosto de 2016 -Fecha de aceptación: 25 de Octubre de 2016

Resumen: La idea principal de este artículo es indagar en los fundamentos teóricopsicológicos que dan origen a los modelos de satisfacción y estudiar las raíces principales de la configuración de la personalidad del individuo, para concebir una propuesta de un modelo de satisfacción genérico que en el futuro pueda ser desarrollado de forma personalizada para distintas entidades, de servicios sociales y del sector privado en general, con una filosofía de servicio al cliente y de mejora continua. La satisfacción del cliente debe de ser tomada como uno de los aspectos primordiales para la medición del cumplimiento de las expectativas formadas por el usuario sobre el servicio o bien que recibe, y considerando que la satisfacción que el cliente obtiene de un producto o servicio siempre será subjetiva, su importancia es vital para la organización, ya que el comprender y manejar las variables correctas según el tipo de bien propuesto deberá generar la fidelidad en el consumo del mismo y su repetición constante. Por esta razón nuestra propuesta de generar un modelo de satisfacción se justifica debido a que el encontrar las variables precisas va a depender de conocer muchos aspectos del producto servicio ofrecido y del comportamiento habitual del cliente que lo utiliza.

Palabras clave: modelo; satisfacción; regulación; estados

Abstract: The main idea of this article is to research the theoretical and psychological foundations that give rise to satisfaction models and study the main roots of the personality settings of the individuals. This study will define a proposal fora generic satisfaction model that in the future can be custom-developed for various entities, social services and the private sectors in general, with a philosophy of customer service and continuous improvement. Customer satisfaction should be taken as one of the key aspects for measuring compliance with the expectations formed by the user on the good or service received and considering that the satisfaction that customers gets of a product or service will always be subjective. Its importance is vital for the organization, because the understanding and managing of the correct variables on the type of product proposed should generate loyalty in the consumption of it and its constant repetition. For this reason, our proposal to build a satisfaction model is justified because the finding of precise variables will depend on knowing many aspects of the product or service offered and the usual customer behavior that uses it.

Key words: model; satisfaction; regulation; states 


\section{Introducción}

Para incursionar en el estudio y análisis de los modelos de satisfacción de clientes, creemos que es muy necesario atender a un enfoque persono lógico del individuo, es decir observar directamente a la estructura de la personalidad del ser humano y este enfoque nos podrá iluminar para estructurar mejores modelos de satisfacción.

Según Bermúdez (1996), existen dos esferas dentro de la estructura de la personalidad del individuo bien claras que son la Motivacional Afectiva y la Cognitiva Instrumental:

"la inductora o motivacional-afectiva, la cual explica, a través de su funcionamiento, el por qué y el para qué de la actuación de la persona, y la ejecutora o cognitivo instrumental, que apunta al cómo y al con qué se realiza dicha actuación”.

La primera está compuesta de la Unidad psicológica llamada Orientación Motivacional que moviliza a la persona a realizar alguna cosa; Está la esfera llamada Expectativa

Motivacional, la cual dirige la actuación de la persona hacia determinado objetivo; y, finalmente tenemos la unidad llamada Estado de Satisfacción, que sostiene a la persona en su actuación, es decir la mantiene interesada.

Por su parte la esfera Cognitivo Instrumental está configurada por los estados Cognitivo, Meta cognitivo y la Instrumentación Ejecutora, donde cada uno tiene su función independiente pero están íntimamente relacionadas e intrincadas fuertemente, de tal manera que es imposible separarlas, sólo para efectos de análisis es que se les distingue por sus funciones.

Lo cognitivo trabaja en la generalización de los conocimientos, mientras que el estado meta cognitivo abarca cualquier manifestación de reflexión o meditación acerca de los propios conocimientos y por su parte la instrumentación ejecutora, comprende las actuaciones de la persona en forma de instrumentaciones conscientes o inconscientes traducidas en acciones, operaciones, habilidades, hábitos y capacidades.

"Sin negar la validez teórico-metodológica de muchas de las teorías o concepciones que sobre la personalidad existen, nosotros consideramos que definir esta categoría como configuración psicológica de la autorregulación de la persona, que surge como resultado de la interacción entre lo natural y lo social en el individuo”. (Bermúdez, 1996) 


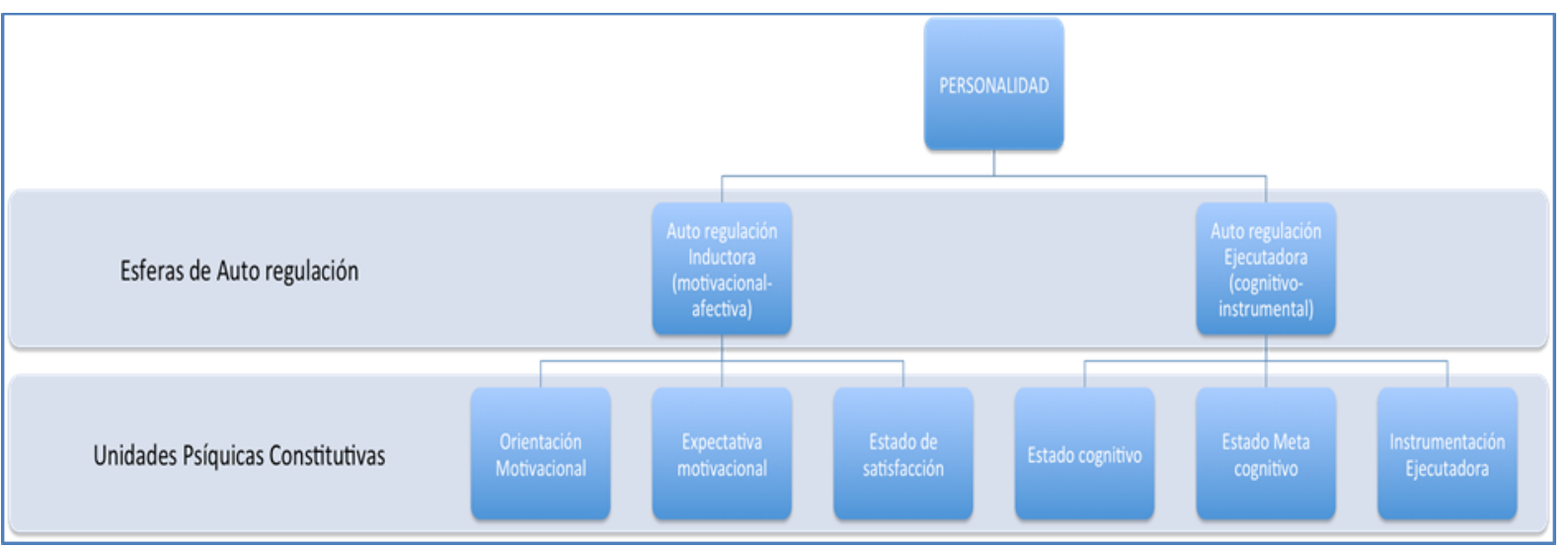

Ilustración 1

\section{Problema}

En qué medida la aplicación de un modelo de satisfacción proporciona las bases para mejorar la calidad del servicio de las organizaciones y como el estudio del mismo generaría aspectos positivos en la percepción de los clientes de los diferentes bienes y servicios.

\section{Importancia del problema}

Es estrictamente necesario al definir un modelo de satisfacción el considerar en primer lugar los elementos de la esfera motivacional-afectiva puesto que son los impulsores de la actuación de la persona y en segundo lugar y no menos importante considerar los estados ya antes mencionados y por supuesto sin dejar de suponer que estos estados y unidades psíquicas están perfectamente interrelacionados y que ninguno se mueve sin el otro.

\section{Objetivo}

Determinar la importancia de la aplicación de un modelo de satisfacción y las variables que pueden afectar a los distintos modelos de negocio.

\section{Hipótesis}

La aplicación variables medibles en un modelo de satisfacción generaría criterios reales de la interacción entre el producto o servicio y la expectativa generada por el cliente.

\section{Justificación}

Imaginemos un cliente que necesita atención médica (o cualquier otro servicio), este requiere conocer qué institución (o profesional) puede brindarle el servicio y tomará una decisión en base a un sin número de factores coyunturales, como beneficios y precios, por citar algunos más comunes. Previo a su decisión, va a buscar la mejor alternativa, entre las que conocen en ese momento, y para ello recurre a la memoria y además busca información de otras fuentes que le resulten confiables, preguntando a otras personas para luego decidirse por aquella alternativa que según los datos obtenidos y evaluados resulte como la mejor opción. Es en esta etapa donde 
ejerce un rol protagónico la metacognición, que regula y evalúa las alternativas que ya tiene en su cabeza dicho cliente y por lo tanto, consigue la orientación que lo motiva a tomar una decisión, la misma que deriva en la generación tácita de la expectativa motivacional correspondiente, que en consecuencia debe ser la expectativa de mayor nivel ya que su elección fue basada en obtener el mayor beneficio posible. Toda vez que el cliente ha contratado el servicio y empieza a usarlo, se acciona el estado de satisfacción y su evaluación instantánea (estado metacognitivo). A partir de aquí se pueden generar tres estados:

a) Desconfirmación positiva de expectativas: Se origina cuando el consumidor que en un principio genera unas expectativas de funcionamiento pre compra (antes de usar el bien) y que luego de utilizar el bien genera percepciones de funcionamiento pos compra (después de usar el bien), decide dentro del nivel de expectativas cubiertas que el producto adquirido sobrepasó las expectativas esperadas y esto origina un grado de satisfacción positivo en el cliente.

b) Confirmación de expectativas: Este modelo concibe la satisfacción como el resultado o contraste entre la realidad percibida por el individuo y algún tipo de estándar de comparación de expectativas o de normas basadas en la experiencia del consumidor.

c) Desconfirmación negativa de expectativas: Se da cuando el bien a utilizar por parte del comprador no cumple con las expectativas mínimas esperadas y eso genera una pobre percepción de la satisfacción del producto utilizado en el cliente. En esta situación hay que tomar en consideración que puede darse el caso que el cliente puede haber tenido muy altas las perspectivas del producto o servicio y al no cumplirlas se genera esta desconfirmación negativa.

\section{Métodos}

Modelo de satisfacción con el enfoque personológico versus modelo tradicional

\section{Modelo tradicional}

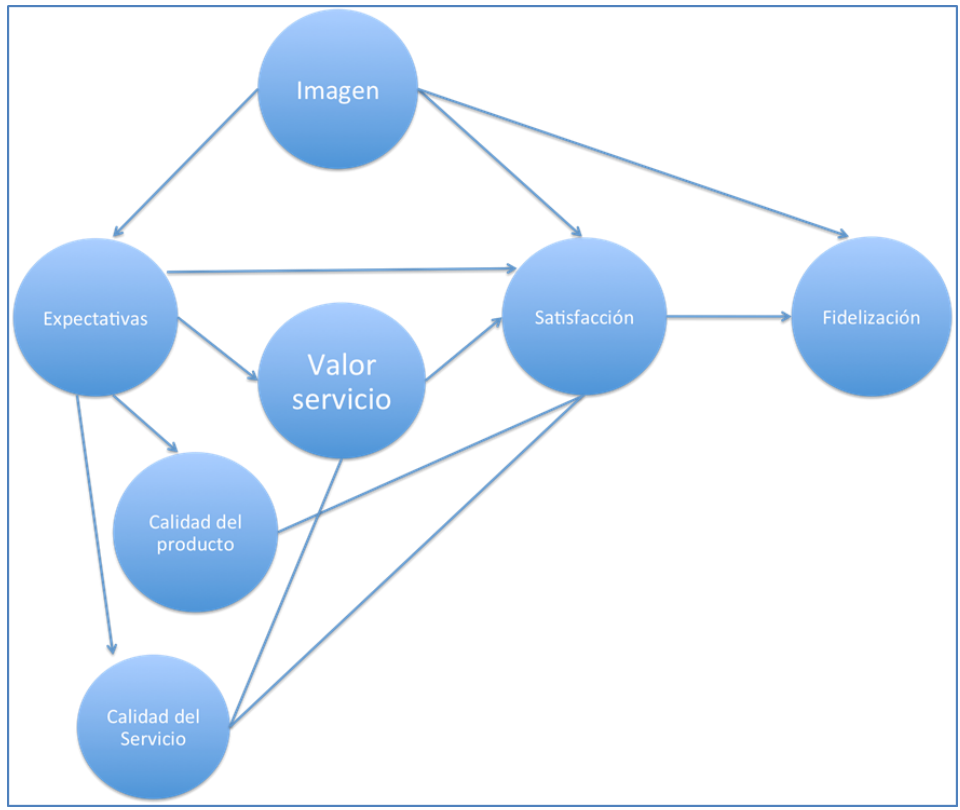




\section{Ilustración 2: Modelo ECSI (Índice Europeo de Satisfacción del Cliente)}

\section{Modelo personológico}

En este gráfico los cuadros azules representan el modelo personológico y los rojos representan el modelo tradicional.

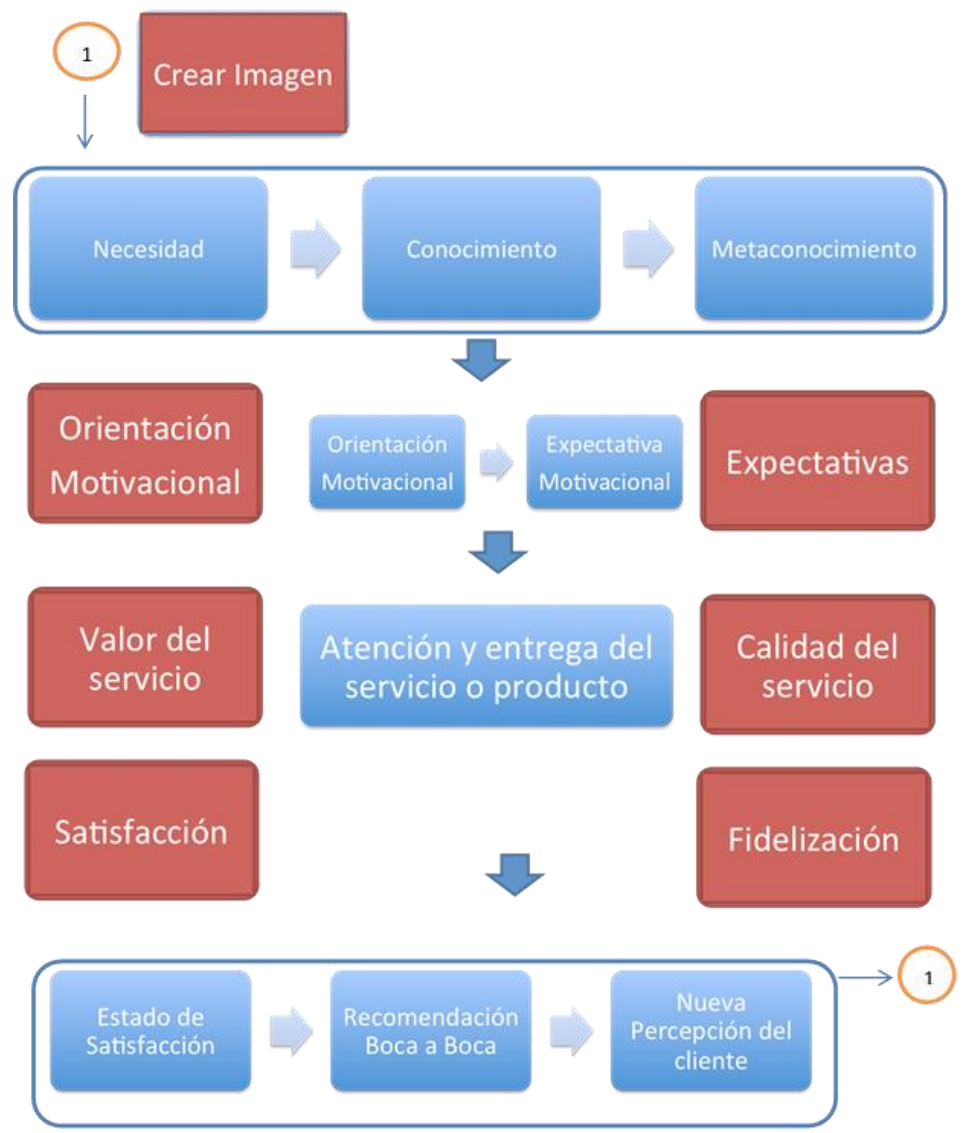

Ilustración 3: Incorporando modelo personológico 


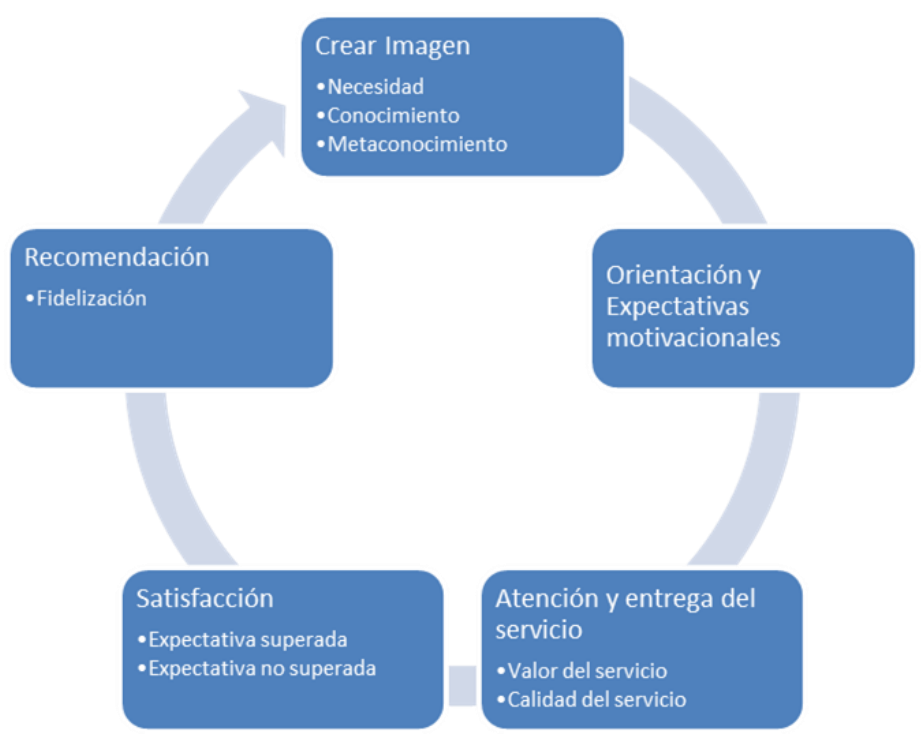

Ilustración 4: Modelo Propuesto

El modelo consiste en tres fases fundamentales: pensemos en un laboratorio médico que realiza exámenes médicos y de oftalmología

Primera fase: Generar en el potencial interesado en recibir servicios, un estado de conocimiento e información acerca de lo que este recibiría si asiste al laboratorio, es decir proveer de alguna manera la información pertinente de lo que el laboratorio sabe y puede hacer. En esta fase el cliente potencial existe y no necesariamente está vinculado con la empresa, por eso se debe generar una campaña de información dentro y fuera de la empresa, donde haya información detallada de tal forma de generar conocimiento y metaconocimiento acerca del servicio.

Segunda fase: En esta fase existen clientes con cierta orientación y con un nivel de expectativas respecto al servicio ofrecido. Es claro que el grado de expectación es directamente proporcional con el grado de decisión al requerir los servicios ofrecidos por la empresa en cuestión.

Tercera fase: Es la decisiva donde se deben llenar todas las expectativas generadas en los potenciales clientes y además se debe superar dichas expectativas con un servicio de calidad, atención amable y entrega hacia el cliente. En esta fase debe haber un acompañamiento al cliente en todo momento que dure el servicio, con atención personalizada cumpliendo con los protocolos de servicio establecidos por la organización. Es aquí donde se puede romper el círculo si la atención y entrega del bien o servicio es deficiente en base a las expectativas generadas en la etapa anterior.

Cuarta fase: Es absolutamente necesario evaluar el estado de satisfacción de los clientes inmediatamente después del servicio, es nuestra última oportunidad ya que no volverá hasta que nos vuelva a necesitar, pero es luego de su partida que comentará su experiencia a otras personas, potenciales clientes y se generará en esa persona una renovada percepción. 
Quinta fase: Si la experiencia fue positiva, el reflejo natural de todo consumidor será el de generar comentarios positivos a sus referidos, al finalizar el ciclo, se vuelve al punto de partida pero con una nueva percepción.

\section{El Modelo de satisfacción del cliente KANO}

Este modelo se centra en que la satisfacción del cliente depende por completo de la capacidad que tiene la empresa de generar su producto o servicio, basándose en que no todas las características de un producto o servicio producen la misma satisfacción en el cliente, sino que algunas de ellas contribuyen en mayor grado a generar una mayor fidelidad. A continuación explicaremos las características agrupándolas en grupos:

- Características-requisitos básicos: son características del producto que el cliente considera obligatorias. No aumentan la satisfacción del comprador, pero causan una insatisfacción muy grande si no se aportan.

- Características-requisitos de desempeño: estas características del producto aumentan proporcionalmente la satisfacción del cliente. Cuantas más se añaden o más funcionalidades ofrecen, más satisfecho está el cliente.

- Características-requisitos de deleite: son características no esperadas por el cliente y que causan una gran satisfacción. Como no son esperadas, no provocan insatisfacción si no se aportan.

Según este modelo, todas las características que tengan alguna influencia directa o indirecta en la satisfacción del cliente se clasificarán en alguno de estos tres grupos antes mencionados.

El modelo de Kano se inserta en la fase tres de nuestro modelo y complementa sustancialmente las características necesarias para mejorarlo.

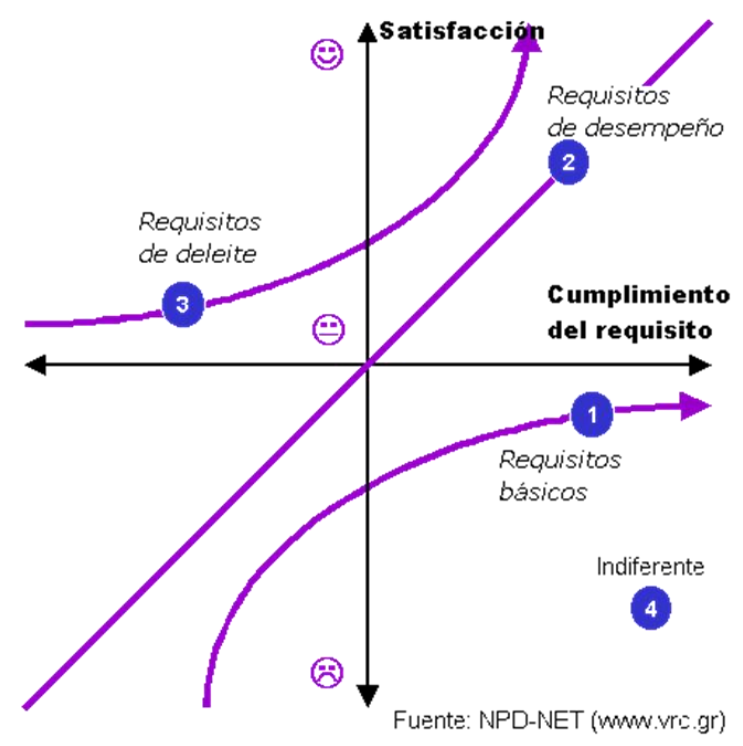

Ilustración 5: Modelo Kano 
A continuación describimos lo que sucede en cada punto del gráfico anteriormente expuesto:

1. Si los requisitos básicos no se aportan al $100 \%$ y en perfectas condiciones, siempre habrá insatisfacción, por pequeña que sea. No es nada fácil obtener elevados niveles de satisfacción actuando sólo sobre este tipo de características.

2. Los requisitos de desempeño evolucionan linealmente con la satisfacción. Desde un cumplimiento cero de estos requisitos y una insatisfacción enorme, podemos ir aumentando la satisfacción conforme vamos proporcionando más y mejores características de este tipo.

3. Los requisitos de deleite siempre sitúan la satisfacción por encima de la situación neutral. A medida que estos van creciendo, la satisfacción aumenta con mayor rapidez de lo que se incrementaría si sólo aportamos al producto o servicio características de desempeño.

\section{Matriz Importancia-Resultado}

En complemento, la matriz importancia-resultado nos permite identificar los aspectos que se pueden analizar dentro de la satisfacción percibida y expectativas latentes del mercado objetivo según lo describen los cuadrantes:

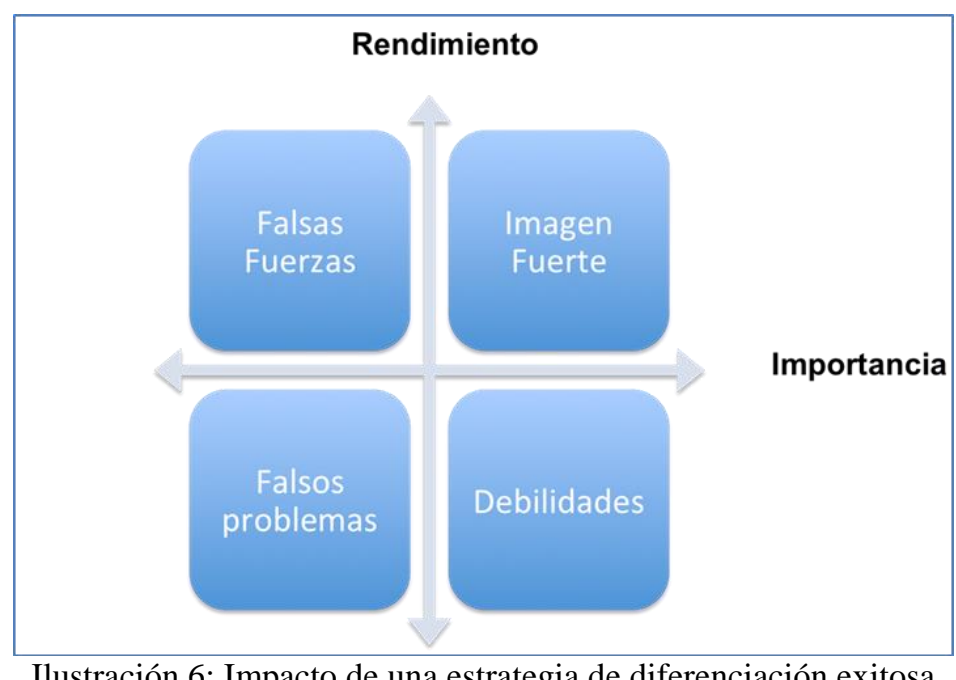

1. Imagen Fuerte: Serán aquellos atributos de alto desempeño que son importantes para el mercado objetivo, en los que la organización debe concentrarse en mantener y mejorar.

2. Debilidades: Serán aquellos atributos de bajo desempeño que son atractivos para el mercado objetivo, en los que la organización deberá concentrarse en mejorar.

3. Falsos Problemas: Serán aquellos atributos de bajo desempeño que no son atractivos para el mercado objetivo, en los que la organización desperdiciaría recursos al atender como prioritarios.

4. Falsas Fuerzas: Serán aquellos atributos de alto desempeño que no son atractivos para el mercado objetivo, en los que la organización podría mantener el nivel sin que eso signifique algo clave para la satisfacción final del consumidor. 
Para la correcta aplicación de la matriz es necesario, previa investigación, definir la proporción o grado de importancia de los distintos atributos o beneficios que espera un mercado específico de un producto o servicio determinado, de tal forma que se pueda asignar un peso representativo a cada uno de ellos y en consecuencia priorizar el desempeño sobre los de mayor peso.

1. Todo producto o servicio se constituye en base a una sumatoria de atributos observados (aquellos que conoce el mercado), los cuales se representarán como $a !, a !, a !, \ldots ., a !$.. Cabe destacar que un producto puede tener varios atributos importantes, pero en algunos casos no todos los atributos son conocidos por el consumidor, los desconocidos se descartan.

2. A su vez, cada atributo está diseñado para agregar valor al producto o servicio bajo estándares de calidad previamente establecidos, que deben generar un nivel de desempeño percibido $(d !)$, entonces se define que

$$
d_{i}=\% v_{i}+\% c_{i}
$$

Donde $\% v$ ! corresponde al grado de valor proporcionado por cada atributo y $\% c$ ! al nivel de calidad del mismo.

3. A cada atributo $a$ ! le corresponde un peso $k$ ! asignado por el consumidor, donde la sumatoria de dicho peso debe ser igual a 1.

$$
\sum_{i=1}^{n} k_{i}=1
$$

4. Por lo tanto, se puede definir el desempeño total como la sumatoria de los desempeños individuales ponderados.

$$
\sum_{i=1}^{n} d_{i}\left(k_{i}\right)=D
$$

5. Siendo que ya se ha definido a la satisfacción como la capacidad de igualar los niveles de desempeño con las expectativas generadas, así como la desconfirmación positiva o negativa, en caso de superar o no cumplir las expectativas, respectivamente; se plantea que

$$
S=D-E\left\{\begin{array}{l}
\mathrm{S}>0 ; \text { Desconfirmación positiva } \\
\mathrm{S}=0 ; \text { Satisfacción } \\
\mathrm{S}<0 ; \text { Desconfirmación negativa }
\end{array}\right.
$$

6. Ahora bien, el desempeño total D, va a estar formado por el desempeño esperado y el no esperado (definido como adicional, que supera la expectativa inicial del consumidor) y definiremos la expectativa básica con la ecuación 


$$
E=\sum_{i=1}^{n} a_{i} k_{i}
$$

Será determinante que dicha expectativa sea medida con una investigación previa para poder completar la ecuación.

$$
S=D_{n e}+D_{e}-E
$$

7. Reemplazando las variables en la ecuación anterior, tendríamos que:

$$
S=\sum_{i=1}^{r} d_{i N E}\left(k_{i N E}\right)+\sum_{i=1}^{n} d_{i E}\left(k_{i E}\right)-\sum_{i=1}^{n} a_{i} k_{i}
$$

Vemos que la satisfacción puede ser medida por tres sumandos fundamentales.

\section{Resultados}

En primer lugar se ha formulado una interrelación entre un enfoque de la personalidad (que manifiesta el comportamiento humano) y los modelos de satisfacción del cliente. Una forma de interpretar y comprender los diferentes estados del cliente cuando consume un producto o servicio. Esto da pie a seguir indagando y encontrando causas y nuevas estrategias para lograr medir la satisfacción del cliente, que como hemos visto, es muy necesaria a la hora de la fidelización.

En segundo lugar, se presentó una formulación que permite en medir el grado de satisfacción, utilizando atributos del producto o servicio detectados por los clientes y se asignó a ello una forma de medida, que de acuerdo a cada producto o tipo de cliente, seguramente tendrá variaciones, pero es sin ninguna duda, se puede detectar cuan satisfechos han quedado los clientes que han consumido un producto o servicio. El proceso de aplicar esta metodología es el mismo para cualquier producto o servicio, sin embargo, si se aplica a un mismo producto los resultados pueden cambiar de acuerdo a que las percepciones de los clientes son cambiantes y varían en el tiempo y a múltiples factores, por eso la aplicación es siempre única para cada producto y momento.

\section{Conclusiones}

En este trabajo hemos conseguido dilucidar a través del enfoque personológico del individuo el porqué de la conformación de la estructura de los modelos de satisfacción y su configuración lógica, que permite estudiar cómo se puede aplicar en diversos escenarios y en diversos tipos de negocio, lo que abre una buena expectativa para la investigación. En este caso se deben definir los atributos que el cliente percibe como importantes, establecer porcentajes de valor y calidad proporcionado por las preferencias de los clientes.

El presente modelo de Medición de Satisfacción Ponderada (MSP) se presenta como una herramienta que permite la medición cuantitativa real del nivel de satisfacción de un determinado mercado sobre un producto o servicio específico. Para su correcta aplicación, más allá de la 
fundamentación teórica explicada anteriormente, es necesario plantear el modelo matemático para cada caso, el mismo que deberá definirse en función de las variables a continuación descritas:

En consecuencia, el nivel de satisfacción podrá ser medido numéricamente mediante la sumatoria de desempeños esperados y no esperados, en contraste con la expectativa previa que tenga el consumidor.

Lo que sigue entonces es poder aplicar el modelo de satisfacción a un determinado negocio o empresa que permita establecer una lógica de trabajo para estandarizar los procesos de aplicación para poder replicarlo en otras áreas de negocio.

\section{Agradecimientos}

A Dios por brindarnos la sabiduría y el permitirnos adquirir el conocimiento. A nuestras familias por ser los pilares y motores de nuestra motivación diaria. A los amigos y colegas que nos alentaron a realizar y culminar este proyecto. Y a la Universidad Santa María por ser nuestro espacio académico donde hemos podido desarrollar esta investigación.

\section{Bibliografía}

Berger, C. E. (1993). Kanós Methods for understanding customer- defined quality. Center for quality Managment Journal, 3-35.

Bermúdez, S. R. (1996). Teoría y metodología del aprendizaje. La Habana: Pueblo y educación.

Marzo, J. C., Martinez-Tur, V., Ramos, J., \& José, P. (s.f.). La satisfacción del usuario desde el modelo de la confirmación de expectativas: respuesta a algunas interrogantes.

Marzo, J. C., Martínez-Tur, V., Ramos, J., \& Peiró, J. M. (2002). La satisfacción del usuario desde el modelo de la confirmación de expectativas: respuesta a algunos interrogantes. Psicothema, 14(4), 765-770. Psicothema, 765-770.

Patterson, P. (1993.). Expectations and product perfomance as determinants of satisfaction for a high involvence purchase. Psychology of Marketing, 449-465.

Patterson, P., Johnson, L., \& Spreng, R. (1997). Modelling in the determinants of customer satisfaction for business to business professional services. Journal of the Academy of Marketing Sciene, 25-4-17. 